\title{
Genetic Control of Iron Bioavailability is Independent from Iron Concentration in a Diverse Winter Wheat Mapping Population
}

\section{Tally Wright}

National Institute of Agricultural Botany

\section{Keith Gardner}

National Institute of Agricultural Botany

Raymond Glahn

Agricultural Research Service

Matthew Milner ( $\square$ matthew.milner@niab.com )

National Institute of Agricultural Botany

\section{Research Article}

Keywords: Iron, bioavailability, Caco-2, MAGIC, wheat, biofortification

Posted Date: January 14th, 2021

DOI: https://doi.org/10.21203/rs.3.rs-142805/v1

License: (c) (i) This work is licensed under a Creative Commons Attribution 4.0 International License. Read Full License 


\section{Genetic control of iron bioavailability is independent from iron concentration in a 2 diverse winter wheat mapping population}

3 Tally I.C. Wright ${ }^{1}$, Keith A. Gardner ${ }^{1}$, Raymond P. Glahn ${ }^{2}$, Matthew J. Milner ${ }^{{ }^{*}}$

$4 \quad{ }^{1}$ NIAB, 93 Lawrence Weaver Road, Cambridge CB3 0LE, UK

$5 \quad{ }^{2}$ USDA-ARS, Robert W. Holley Center for Agriculture and Health, Ithaca, NY 14853, USA

$6 \quad *=$ To whom correspondence should be addressed, matthew.milner@niab.com.

\section{Abstract:}

8 Background: Anemia is thought to affect up to 1.6 billion people worldwide. One of the major contributors to low iron $(\mathrm{Fe})$ absorption is a higher proportion of cereals compared to meats and pulse crops in people's diets. This has now become a problem in both the developed and developing world, as a result of both modern food choice and food availability. Bread wheat accounts for $20 \%$ of the calories consumed by humans and is an important source of protein, vitamins and minerals meaning it could be a major vehicle for bringing more bioavailable Fe into the diet.

Results: To investigate whether breeding for higher concentrations of Fe in wheat grains could help increase $\mathrm{Fe}$ absorption, a multiparent advanced generation intercross (MAGIC) population, encompassing more than $80 \%$ of UK wheat polymorphism, was grown over two seasons in the UK. The population was phenotyped for both Fe concentration and Fe bioavailability using an established Caco-2 cell bioassay. It was found that increasing Fe levels in the grains was not correlated with higher Fe bioavailability and that the underlying genetic regions controlling grain Fe levels do not co-localise with increased $\mathrm{Fe}$ absorption. Furthermore, we show that phytate levels do not correlate with $\mathrm{Fe}$ bioavailability in our wheat population and thus phytate-binding is insufficient to explain the lack of correlation between Fe bioavailability and Fe concentrations in the wheat grain. Finally, we observed no (Fe bioavailability) or low (Fe concentration) correlation between years for these traits, confirming that both are under strong environmental influence.

Conclusions: This suggests that breeders will have to select not only for Fe levels directly in grains, but also increased bioavailability. 
Iron $(\mathrm{Fe})$ deficiency in humans, also known as anemia, is estimated to effect more than 1.6 billion people worldwide with major implications for many aspects of human health [1]. In places where people's diet is largely cereal-based, anemia is prevalent, mainly due to the low bioavailability of the Fe in cereals relative to diverse Fe sources such as meat and pulse crops [2,3]. While much effort has been expended to try and remedy the large number of cases of Fe deficiency globally, anemia is not just a problem in the developing world. It has recently been estimated that more than half of adolescent girls in the UK aged between 11 and 18 years old are also currently anemic [4]. In many developed nations Fe supplementation programs have been in place for several years, for example in the UK a fortification program has been in place since the early fifties. This fortification requirement requires all flours processed in the UK meet the level of $\mathrm{Fe}>1.65 \mathrm{mg} / 100 \mathrm{~g}$ of flour. The rationale behind the fortification effort is the high penetration of wheat into an estimated $99 \%$ of households in the UK [5]. Nevertheless, high levels of anemia persist in industrialised nations even with these programs in place [4]. The reason for the failure of these efforts is thought to be down to the form in which $\mathrm{Fe}$ is currently added to fortify flours [6]. As most countries use non-recommended, low-bioavailability, atomized, reduced or hydrogen-reduced iron powders to supplement the bread flour [6]. One current strategy for addressing the problem of iron deficiency in humans is through biofortification, with plant breeding or genetic engineering techniques being used to produce new varieties of staple foods with higher iron content in major crops species such as rice, wheat, maize, millet, and legumes. As transgenic foods do not share the same consumer acceptance as traditionally bred varieties, 'naturally' bred biofortified crops have been the preferred route to increase Fe intake regardless of GM regulation and public acceptance [7-9].

Substantial natural genetic variation of $\mathrm{Fe}$ content of wheat grains has been identified in bread wheat and its progenitors, with Fe levels ranging from 19 to $71 \mathrm{mg} / \mathrm{kg}$ [10-12]. This variation is currently being exploited to select iron-rich genotypes for biofortification with the hope to improve iron absorption from the grain [13,14]. Furthermore, recent attempts to fortify different fractions of the grain suggest that both whole grain and endosperm levels of Fe can be improved both by transgenic means and more traditional breeding [15]. One large problem with this strategy is while Fe concentration in the grain can be improved genetically by breeding for higher Fe levels, this rarely translates to increased $\mathrm{Fe}$ absorption by humans $[14,15]$. There has been some advancement in the increased concentration and bioavailability of Fe using transgenic means, but it remains to be seen if these varieties will be adopted by a larger consuming public [16-18].

The underlying reason for poor Fe bioavailability from cereals is complex, as the reactive nature of this trace mineral enables strong interactions with other components of the grain that affect $\mathrm{Fe}$ bioavailability. Phytate and certain polyphenols and phenolic acids are major components in plant foods that effect Fe absorption [19,20]. Fifty to sixty percent of the iron in cereal grain is bound to inositol 
hexakisphosphate (IP6) or pentaphosphate (IP5) and forms phytate salts in the aleurone layer of the grain and germ [20,21]. This is believed to be the main reason for low Fe bioavailability in wheat as the molar excess of phytate complexes the Fe and limits exchange of luminal Fe to the iron transporter, thus preventing absorption in the gut $[22,23]$. In addition to phytate, the aleurone layer of grains, is known to contain polyphenols and phenolic acids the majority of which in wheat are phenolic acids, which can both promote and inhibit absorption of Fe by the gut $[19,24]$. It has been suggested that wheat may not contain or produce many of these polyphenols and/or that large environmental effects can drastically change the relative amounts of these compounds in the grain and thus might be one of the underlying reasons for the lower reported values of Fe bioavailability in wheat [24-26]. Also more recently different fractions of the grain itself have been shown to inhibit absorption of Fe from grains and can account for a fivefold difference in Fe concentration of the material versus actual $\mathrm{Fe}$ absorption [27].

The above factors are the primary reasons why simply increasing Fe intake alone does not always result in more $\mathrm{Fe}$ absorption; thus, to properly assess the nutritional quality of $\mathrm{Fe}$ in foods, primarily due to the chemical nature of Fe and the degree to which phytochemicals can bind Fe and be present in high molar excess.

While most of the studies presented so far shed light on the mechanisms of bioavailability of Fe from grains, none have investigated the genetic architecture of this complex trait. In order to do so, one must address both $\mathrm{Fe}$ concentration and Fe bioavailability together and alone can help increase delivery of $\mathrm{Fe}$, or if bioavailability is a different trait to breed for, is to evaluate both traits in a diverse mapping population and map the quantitative trait loci (QTL) underlying them. Here we use an 8-founder MAGIC (Multiple parent Advanced Generation Inter Crossing) population, encompassing more than $80 \%$ of the genetic polymorphism of UK bread wheat [28], to identify QTL underlying both Fe grain concentration and bioavailability. The population was screened for bioavailability using an established Caco-2 cell bioassay for Fe bioavailability. This model provides a relative measure of absorbable $\mathrm{Fe}$ from a given amount of sample, thus providing a practical measure of Fe delivery. In the present study, both Fe concentration and Fe delivery (ie. bioavailability) were measured from samples collected from two separate field seasons, to identify the stability as well as the possible colocalisation of QTL for both Fe concentration and bioavailability.

\section{Materials and Methods:}

\section{Field experiment:}

Seed were sampled from 1100 MAGIC recombinant inbred lines grown in randomised $1 \mathrm{~m}^{2}$ nursery plots during the 2015-2016 ("year 1") and 2016-2017 ("year 2") field seasons at the NIAB experimental farm in Cambridge, UK, using the agronomy package detailed in Suppl. Table 1. In year 1, 244 
independent BC1F8 offspring lines plus all of the eight founder lines of the MAGIC population were measured for both Fe content and Fe bioavailability. In year 2, 288 independent BC1F9 offspring lines were measured, including all lines from year 1 but only two of the eight founder lines. Twenty grams of seed from each line were dried and milled using a hammer mill (Glen Creston) with a $1 \mathrm{~mm}$ sieve for use in later experiments.

\section{ICP-AES:}

Fe content of all samples was conducted via inductively coupled plasma emission spectroscopy (ICPAES). For each sample $0.5 \mathrm{~g}$ flour was dried down and then treated with $3.0 \mathrm{~mL}$ of $60: 40 \mathrm{HNO}_{3}$ and $\mathrm{HClO}_{4}$ mixture in a Pyrex glass tube and left overnight to destroy organic matter. The mixture was then heated to $120^{\circ} \mathrm{C}$ for two hours and $0.25 \mathrm{~mL}$ of $40 \mu \mathrm{g} / \mathrm{g}$ yttrium added as an internal standard to compensate for any drift during the subsequent inductively coupled plasma atomic emission spectrometer (ICP-AES) analysis. The temperature of the heating block was then raised to $145^{\circ} \mathrm{C}$ for two hours. Then, the temperature of the heating block was then raised to $190^{\circ} \mathrm{C}$ for ten minutes and then turned off. The cooled samples in the tubes were then diluted to $20 \mathrm{~mL}$, vortexed and transferred into auto sample tubes to analyze via ICP-AES. The model of the ICP used was a Thermo iCAP 6500 series (Thermo Jarrell Ash Corp., Franklin, MA, USA). Three technical replicates were taken for all lines tested.

\section{Bioassay for Fe Bioavailability:}

Whole grain milled wheat flour samples were subjected to simulated gastric and intestinal digestion as per established bioassay conditions [29]. Intestinal digestion is carried out in cylindrical inserts closed on the bottom by a semipermeable membrane and placed in wells containing Caco- 2 cell monolayers bathed in culture medium. The upper chamber was formed by fitting the bottom of a Transwell insert ring (Corning) with a $15000 \mathrm{Da}$ molecular weight cut-off (MWCO) membrane (Spectra/Por 2.1, Spectrum Medical, Gardena, CA). The dialysis membrane was held in place using a silicone ring (Web Seal, Rochester, NY). Iron uptake by the Caco-2 cell monolayers was assessed as previously described and by measuring ferritin concentrations in the cells [29]. The cells were maintained in Dulbecco's modified Eagle medium plus 1\% antibiotic/antimycotic solution, $25 \mathrm{mmol} / \mathrm{L}$ HEPES and $10 \%$ fetal bovine serum. Forty-eight hours prior to the experiment, the growth medium was removed from the culture wells, the cell layer was washed and the growth medium was replaced with minimum essential medium (MEM) at $\mathrm{pH}$ 7.0. The MEM was supplemented with $10 \mathrm{mmol} / \mathrm{L}$ PIPES, $1 \%$ antibiotic/antimycotic solution, $4 \mathrm{mg} / \mathrm{L}$ hydrocortisone, $5 \mathrm{mg} / \mathrm{L}$ insulin, $5 \mu \mathrm{g} / \mathrm{L}$ selenium, $34 \mu \mathrm{g} / \mathrm{L}$ triiodothyronine and $20 \mu \mathrm{g} / \mathrm{L}$ epidermal growth factor. This enriched MEM contained less than $80 \mu \mathrm{g}$ Fe/L. All ingredients and supplements for cell culture media were obtained from GIBCO (Rockville, MD). The cells were used in the Fe uptake experiment at 13 days post-seeding. In these conditions, the amount of cell protein measured in each well was highly consistent between wells. On the experiment 
day, $1.5 \mathrm{~mL}$ of the digested sample was added to the inserts' upper chamber and incubated for two hours. Then, the inserts were removed and $1 \mathrm{~mL}$ of MEM was added. Cell cultures were incubated for 22 hours at $37^{\circ} \mathrm{C}$.

The protocols for Caco- 2 cell ferritin and cell total protein content analyses were described previously [29]. Briefly, growth medium was removed from the culture well by aspiration and the cells were washed twice with a solution containing $140 \mathrm{mmol} / \mathrm{L} \mathrm{NaCl}, 5 \mathrm{mmol} / \mathrm{L} \mathrm{KCl}$ and $10 \mathrm{mmol} / \mathrm{L} \mathrm{PIPES}$ at $\mathrm{pH}$ 7.0. The cells were harvested by adding an aliquot of deionized water and placing them in a sonicator (Lab-Line Instruments, Melrose Park, IL). The ferritin and total protein concentrations were determined on an aliquot of the harvested cell suspension with a one-stage sandwich immunoradiometric assay (FER-IRON II Ferritin Assay, Ramco Laboratories, Houston, TX) and a colorimetric assay (Bio-Rad DC Protein Assay, Bio-Rad, Hercules, CA), respectively. Caco-2 cells synthesize ferritin in response to increases in intracellular Fe concentration. Therefore, we used the ratio of ferritin/total protein (expressed as ng ferritin/mg protein) as an index of cellular Fe uptake.

Each plate of samples were run on a 6 well plate with internal controls, consisting of a lentil flour sample, ascorbic acid plus $\mathrm{FeCl}_{2}, \mathrm{FeCl}_{2}$, and MEM media alone. In addition, the eight founder lines were used as overlapping controls across days (1-2 founders per day). For year 2, the founder Claire was run as a control on every plate in addition to the other controls. Finally, three technical replicates of all lines and controls were run on each day.

Phytate measurements: Phytic acid was measured using the Megazyme Phytic Acid Assay Kit (Brey, Ireland) according to the manufacturer's directions. The only change was approximately $100 \mathrm{mg}$ of each flour was digested in $1.8 \mathrm{~mL} \mathrm{HCl}(0.66 \mathrm{M})$ in $2.2 \mathrm{~mL}$ tubes, placed in a rotator mixer with a constant rpm of 20 overnight at room temperature rather than the full $1 \mathrm{~g}$ sample suggested. Each sample had three distinct flour samples taken through the whole process to determine phytate amounts for each line tested.

\section{Data analysis}

The grain Fe concentration readings were plotted and visually inspected, there were some extreme outliers present, typically over $80 \mathrm{ppm}$. High readings were inspected and if a measurement was substantially different to the other technical replicates taken from the same sample or displayed high $\mathrm{Al}$ and $\mathrm{Ti}$ grain concentration it was removed from the data. These readings were probably soil contaminants. The absolute means were calculated from the three technical replicates of each line and used in the QTL analysis. In year 1, there were four lines with two replicated samples. From these replicates a generalised heritability $\left(H^{2}\right)$ was calculated on a line mean basis, implemented by the VHERITABILITY function in Genstat [VSN International. Genstat for Windows, 19th ed.; Hemel Hempstead, UK], which uses an estimation of $H^{2}$ proposed by Cullis et al. [30]. 
To account for the large day-by-day measurement variation in Fe bioavailability, a lentil flour sample was included as a standardised positive internal control (IC1) on each measurement date in year 1 . To improve this adjustment in year 2, two controls were included on each measurement date: a durum wheat flour sample was used as the standardised internal control (IC2) and the MAGIC founder 'Claire'. Mixed effects linear models using combinations of the different controls as random and fixed factors, and response scaling models where the daily average of the internal control was subtracted from the measurements of corresponding MAGIC lines, were compared in Genstat using Akaike Information Criterion and significance tests of the sample (genotype) effect. Although variance between technical replicates was typically low across both years, in year 2 there were some outliers with high variance between the technical replicates. The individual readings that contributed to the high variance between these technical replicates were removed. In year 2, three genotypes and one internal control were removed which had large variance across all replicates, suggesting possible contamination. The Best Linear Unbiased Predictions (BLUPs) were extracted from the best models and used for subsequent analysis. In year 1, the corrected means of two genotypes with very high ng ferritin/mg protein readings were removed from the dataset as possible contaminants. Cross-trait and cross-year correlations across the population were estimated in R [31] using the Pearson correlation coefficient.

A Shapiro-Wilk test of normality was used to inspect trait distributions and subsequently, QTL mapping was carried out within R using 7367 unique mapped SNP markers from the Illumina Infinium iSelect 90K SNP wheat array [32] as described in Gardner et al [33]. Three analysis approaches were used for QTL detection: single marker regression using R/lme4 (IBS, [34]), interval mapping in R/mpMap (IM, [35]) and composite interval mapping with up to three covariates using R/mpMap (CIM). For IBS, two methods of adjustment for multiple-test correction were used. Firstly, a standard multiple-test correction was carried out in $\mathrm{R}$ using a False Discovery rate (FDR) correction with a threshold of $p<0.05$. A second less stringent method used a Bonferroni significance threshold of $-\log (10)=3.68$, based on $\alpha=$ $0.05 / 237$, where the denominator is the estimated average haplotype number per line in the population, based on map length and number of generations of recombination events. This $2^{\text {nd }}$ method takes into account that markers within haplotypes are highly correlated. For the IM/CIM analyses, an initial liberal cut-off of $-\log 10 \mathrm{p}<3$ and a window size of 100 markers was used in the mpMap function 'findqtl'. The mpMap function 'fit' was then applied, and QTL retained which had $p<0.05$ in the fitted model, as well as percentage variation explained $>1 \%$. In year 1, 237 and 235 MAGIC individuals were used for the QTL analysis of Fe concentration and bioavailability, respectively. In year 2, 284 individuals were used for both traits.

Power analyses were completed with the genotype data of the 235 individuals from year 1 , using a custom R script. A single marker was randomly taken as the site of a focal QTL with 100 other markers on other chromosomes used as minor QTL. The power analyses were completed with four percentage variations explained by the focal QTL $(5,10,25$ and 50\%), with the remaining variation shared across 
the 100 minor QTL. For each percentage variation, 1000 random phenotypes were simulated for each of five heritability values $(0.15,0.25,0.50,0.75$ and 0.90$)$, achieved by adding random normal variation relative to each heritability. Interval mapping in $\mathrm{R} / \mathrm{mpMap}$ was then completed with each simulated phenotype, using the same thresholds listed above. A positive detection was recorded when the focal QTL fell within $20 \mathrm{cM}$ of a significant QTL peak.

\section{$\underline{\text { Results: }}$}

\section{Phenotypic Analysis}

\section{Fe concentration:}

The absolute means were taken from the three technical replicates and used as the phenotypic data for grain $\mathrm{Fe}$ concentration. Observed grain Fe concentrations in the MAGIC population lines ranged from 20.4 to $44.2 \mathrm{ppm}$ in year 1 and 21.7 to $47.9 \mathrm{ppm}$ in year 2 . Very similar means were observed across the years (year $1=32.8$ and year $2=32.3 \mathrm{ppm}$ ). Once the erroneous measurements had been removed, both distributions appeared to be normal (Figure 1; year 1: $W=0.99, P=0.11$; year 2 : $W=0.99, P=$ 0.15). In year 1, the founder Fe concentrations varied only from 28.2 (Alchemy) to $37.2 \mathrm{ppm}$ (Robigus), suggesting that there was substantial segregation distortion in the population (Figure 1A). However, one of the two founders measured in year 2, Claire, had an Fe concentration of only 21.7, which fell in the lowest 3\% of the population (Figure 1B). The other founder measured in year 2, Robigus also showed a considerably different Fe concentration compared to year 1 . Furthermore, there was a low $H^{2}$ observed for grain Fe concentration in year $1\left(H^{2}=0.19\right)$. These trends indicated either the measurement variance of the trait was high, and/or the Genotype $x$ Environment (GxE) interaction was large between years. However, there was a significant correlation between Fe concentrations in year 1 and year 2 across the whole population $(\mathrm{r}=0.27, p<0.01$, Figure $1 \mathrm{E})$.

\section{Bioavailability:}

It was observed that there were very large differences between the means of the MAGIC lines for each Caco-2 plate run. To try and normalise for plate variation, a single internal control was included in year 1 and then two controls were included with the year 2 field season samples on each day (Internal control 2 (IC2) and Claire). It would be expected that the controls should follow the same pattern as the line means for each date of measurement, assuming random lines were used each day. In year 1, the line means weakly tracked with the IC1 means. In year 2, line means followed trends in the controls on most 
days (Suppl. Figure 1) but the IC2 readings were considerably higher than either Claire or the line means.

In year 1, the model which worked best to smooth the large day-by-day measurement variation included the IC1 measurements in the data, with measurement date and genotype treated as random factors. Using this model, $H^{2}$ was calculated as 0.20 for bioavailability. In year 2 a similar approach was followed: the daily measurements of the controls Claire and IC2 (scaled to have the same mean as Claire) were included in the data, with genotype and measurement date treated as a random factors. In year $2, H^{2}$ was higher and estimated as 0.84 . It should be noted that 'heritability' is only in the context of the experimental set up for measuring bioavailability: all replicated samples in the experiment came from the same field plot. From the two models fitted for each year, the best linear unbiased predictions (BLUPs) were extracted and used in the analysis. The use of two controls in the second year (Claire and the scaled IC2) clearly improved the model.

In year 1, bioavailability ranged from 2.7 to $8.1 \mathrm{ng} / \mathrm{mg}$ total protein, with a mean of $5.5 \mathrm{ng}$ ferritin/mg protein (Table 1). Robigus was the founder with the lowest bioavailability (5.2 ng ferritin/mg protein), while Claire showed the highest (7.9 ng ferritin/mg protein), again suggesting the population shows substantial transgressive segregation for this trait. For year 2, bioavailability showed a much greater range from 3.0 to 24.3 with a mean of $10.9 \mathrm{ng}$ ferritin $/ \mathrm{mg}$ protein, considerably higher than in year 1 (Table 1, Figure 1D). Furthermore, in year 2 Claire had a marginally different bioavailability of $9.4 \mathrm{ng}$ ferritin/mg protein, whereas there was a substantial increase to $11.9 \mathrm{ng}$ ferritin/mg protein in Robigus. The Shapiro-Wilk test of normality indicated that both distributions were non-normal in distribution $(P$ $<0.01$ ); there was a slight left skew in year 1 and a more pronounced right skew in year 2 , although we concluded the data skews did not warrant data transformation for QTL mapping. Bioavailability was not significantly correlated between the BLUPs from the different years (Figure 1E). Furthermore, heritability of models including both years was low. This suggests the inter-annual field environmental variance dwarfs the genotypic effects and/or variance due to measurement error is much larger than genotypic effects. In the latter case, the effect appears to be stronger for the year 1. Furthermore, no significant correlation was detected between bioavailability and iron concentration in either year (Figure $1 \mathrm{E})$.

\section{Role of Phytate as an explanation of variation of Fe bioavailability of wheat:}

A lot of attention has been paid to the role which phytate plays in Fe absorption. To understand if differences in Fe content and if phytate levels in the grain could explain some of the differences seen in the bioavailability between lines, phytate was also measured on the population founders from year 1 . The phytate levels varied from $0.7880 \mathrm{~g}$ of phytate per $100 \mathrm{~g}$ of flour in Brompton to $1.237 \mathrm{~g}$ of phytate per 100g flour in Claire (Suppl. Table 2). There was no trend between phytate levels and bioavailability but, when phytate level was plotted against ng ferritin produced from the same flour a slight positive 
correlation was observed (Figure 2), although this was not significant ( $p=0.61$ ). If bioavailability is normalised by the amount of $\mathrm{Fe}$ also present in flour (i.e., the molar ratio of phytate to $\mathrm{Fe}$ ) a clear but still not significant trend was observed ( $p=0.21$, Figure 2$)$. Nevertheless, these results suggest that phytate alone does not explain the differences seen in the bioavailability of Fe of the lines tested.

\section{QTL mapping}

\section{Power analysis}

The results from the power analyses are shown in Suppl. Figure 2. The probability of finding QTL increased with higher percentage variation explained by the focal QTL and higher heritability associated with the simulated phenotypes. Bioavailability and Fe concentration had an estimated $H^{2}$ close to 0.2 in year 1 . With a trait heritability in this region, the power analyses indicated the probability of finding a QTL explaining 50\% phenotypic variation was less than $25 \%$, while finding a more minor QTL that explained $5 \%$ variation had a probability of less than $5 \%$. In year 2, the estimated $H^{2}$ of bioavailability increased to 0.84 . At the higher heritability the probability of finding a QTL that explained $50 \%$ phenotypic variation increased to close to $100 \%$, while the probability of finding a minor QTL (explaining $5 \%$ variation) was around $20 \%$. Our heritability estimates in this study were not very precise due to low numbers of reps. Therefore, the true detection power probably lies in between these two extremes.

\section{Fe concentration}

Across both years, five QTL were identified using IM across five chromosomes (Table 2, Figure 3), although none co-located between the years. Using CIM, five QTL were mapped to the same chromosomes and an extra QTL was mapped to $29.5 \mathrm{cM}$ on $2 \mathrm{~B}$ in year 1. The five QTL found through both IM and CIM were approximately mapped to the same location, excluding the QTL on 3D that was mapped to $46.8 \mathrm{cM}$ using IM and $180.1 \mathrm{cM}$ using CIM.

For year 1, QTL were found on chromosomes 2B, 3D, 5D and 6A (Table 2, Figure 3). The most significant of these hits was present on 2B $(217.5 \mathrm{cM})$. For this QTL, the Xi19 and Rialto haplotypes had the most positive effect and the Brompton and Hereward haplotypes had the most negative effect on Fe levels in the grain. Also in year 1, a QTL was mapped to this same region through the IBS method, the peak marker was found at $220.7 \mathrm{cM}$ on $2 \mathrm{~B}$ with a $-\log 10(P)$ of 3.95 (Table 3). For the other QTL on $2 \mathrm{~B}(29.5 \mathrm{cM})$ and the single QTL on $6 \mathrm{~A}$, the Xi19 haplotypes also had the most positive effect on Fe levels. For the QTL identified on 5D in the CIM approach, Xi19 also contributed the most positive effect, although this was not consistent with the IM approach. The QTL identified on 5D through IM and CIM, was mapped to 175.5 and $199.08 \mathrm{cM}$, respectively. For these QTL, the Rialto haplotype 
contributed the most negative effect on the trait, followed by Claire in the CIM, and Claire and Robigus in the IM. Through the IBS method a QTL was also found on 5D at $181.1 \mathrm{cM}$ with a $-\log 10(P)$ value of 4.55 (Table 3), at the peak marker (BS00032035_51) the founders Rialto, Claire and Robigus all shared the same allele, indicating this was the same QTL found in the IM and CIM. For the 3D QTL identified using IM, Rialto also contributed the most negative effect on the trait. However, for the QTL found through CIM on 3D, Rialto contributed a more positive effect indicating that these loci may be different QTL. No percentage variation explained by a QTL was greater than $10 \%$. The highest percentage variation explained by a QTL was identified was for the QTL mapped to 6A (8\%) where again the Rialto haplotype contributed the most negative effect on the trait.

In year 2, no significant QTL were found for the IBS mapping and only a single QTL was found with the IM and CIM approaches: on chromosome 2A, with the Brompton and Xi19 haplotype having the most positive effect and the Soissons and Robigus haplotype the most negative effect on $\mathrm{Fe}$ concentration. This QTL explained 6.3\% of the trait variation. Overall, for the Fe concentration QTL mapped in year 1 and 2, the Xi19 haplotype typically contributed to the most positive effect on the trait, while the Robigus and Rialto haplotypes typically had a negative effect. This pattern was not observed in the phenotypic variation in founders (Figure 1A and B) where Robgius had the highest $\mathrm{Fe}$ concentration across both years, although only two founders were measured in year 2 .

\section{Fe bioavailability}

Fewer QTL were found for bioavailability than Fe concentration. Furthermore, there were no co-located QTL for both traits and the QTL profiles are quite different (Figure 3) For IM in year 1, two QTL were found on chromosome 1A with peaks at 167.3 and $193.7 \mathrm{cM}$ (Table 2). The QTL profiles shown in Figure 3 indicates that these two peaks were linked to the same QTL due to the presence of a long and messy peak along a considerable proportion of $1 \mathrm{~A}$, although it should be noted that the parental effects are not consistent between the QTL (Table 2). However, as there is no significant correlation between the years, the accuracy of determining parental effects might be speculative. The most significant of the IM hits on 1A was mapped to $194 \mathrm{cM}$, close to the same hit that appeared through the CIM with a peak at $195 \mathrm{cM}$. At this locus the Claire haplotype had the largest positive effect on $\mathrm{ng}$ ferritin/mg protein and the Brompton haplotype had the most negative effect. There were also two other QTL detected on chromosomes 2A and 7B through CIM in year 1. These QTL explained a lower percentage of the phenotypic variance than the 1A QTL (Table 2). An additional QTL was found through IBS mapping on 5B (Table 3), which was not found through IM and CIM.

In year 2, two significant QTL were identified using CIM. One QTL was mapped to $10.5 \mathrm{cM}$ on $2 \mathrm{~A}$ and explained $4.5 \%$ of phenotypic variation. At this QTL, the Alchemy and Hereward haplotype 
contributed the most negative effect on the trait. This QTL was also mapped through the IBS method with a slightly different peak of $18 \mathrm{cM}$ on $2 \mathrm{~A}$ (Table 3), at the peak marker (Excalibur_rep_c110303_320) Alchemy and Hereward shared the alternative allele to the other founders. The second QTL found through CIM in year 2 was mapped to $55.7 \mathrm{cM}$ on $4 \mathrm{~B}$ and explained $4.4 \%$ of phenotypic variation (Table 2). The QTL mapped to $2 \mathrm{~A}$ for Fe bioavailability in year 1 is not likely to be the same as the 2A QTL in year 2 . They are $77 \mathrm{cM}$ apart and in year 1, the Rialto founder haplotype contributed the most negative effect on the trait, whereas for the year 2 QTL, Rialto contributed the third most positive effect on the trait. A QTL for Fe concentration in year 2 was also mapped on 2A, but this QTL was located at the other end of the chromosome $(253 \mathrm{cM})$. Therefore, all three of these QTL are most likely different loci.

\section{$\underline{\text { Discussion: }}$}

Increased $\mathrm{Fe}$ concentration has been suggested to be a major breeding target of improved nutrition for humans in crops [36-43]. However, it is important to note that the target of increased Fe concentration assumes that more $\mathrm{Fe}$ will be delivered for absorption. Given the chemical nature of $\mathrm{Fe}$ and it's interaction with phytochemicals such as phytate, phenolic acids and polyphenols, recent studies now show that it is essential to also evaluate the delivery of Fe (ie. Fe bioavailability) simultaneously with Fe concentration $[44,45]$.

Thus, our goal was to understand how higher grain Fe concentration in wheat could play a role in increased iron absorption/bioavailability. We measured both Fe content and absorption from two field seasons in $>200$ lines of a highly diverse mapping population. A relatively weak $(r=0.27)$ correlation was observed across years for Fe concentration in the current study. It is possible that this could partially be a result of high measurement variation for the trait. However, we successfully identified four QTL explaining around $30 \%$ of the genetic variation of Fe concentration in total in year 1, and these differed from the single QTL found in year 2, with the QTL profiles between years being noticeably different (Figure 3). Therefore, we conclude that there is a high level of Genotype x Environment (GxE) interaction for Fe levels in the grain, which may have had an impact on the success of breeding for increased Fe concentration [11,39,41,46,47], despite the evidence of underlying QTL variation in a number of important cereal species $[11,16,41,46,48,49]$. However, it should be noted that the power analyses (Suppl. Figure 2) highlighted that the chances of finding a QTL linked to a phenotype with a $H^{2}$ of 0.2 was lower than $30 \%$ for all the tested percentage phenotype variations explained by a QTL. For Fe concentration, $H^{2}$ was estimated as 0.19 in year 1, meaning the probability of finding consistent minor QTL over multiple years was very low. The QTL identified here are different than previous studies in wheat using a biparental population being currently grown in Mexico by CIMMYT, or QTL found in a bread wheat progenitor $[13,48]$. 
The day-to-day variation in the Caco-2 assay for bioavailability presents significant analytical challenges and our year 1 data had insufficient well-distributed controls to accurately estimate trait means across the assays $\left(H^{2}=0.20\right)$. We were able to improve this in year $2\left(H^{2}=0.84\right)$ but would recommend that more controls (at least three control lines run on every day in addition to the internal control) be used for this system if testing large numbers of samples. There was no correlation between the bioavailability scores across the two years, again suggesting possibly high GxE variation for this trait, although the low accuracy of the year 1 trait mean estimation is likely to have been a significant factor in the lack of a between-year correlation. It is also notable that the range of bioavailability scores within the population was very different between the two years (2.7-8.1, mean $5.5 \mathrm{ng}$ ferritin/mg protein in year 1, 3.0-24.3, mean $10.9 \mathrm{ng}$ ferritin/mg protein in Year 2). Furthermore, the increased variability with higher trait values (e.g. for control IC2 seen in Suppl. Figure 1) suggests that the measurement error may not scale linearly, which would further negatively impact between-year correlation. Nevertheless, a small number of weak QTLs were detected in both years, albeit explaining a relatively low total percentage of the variation. The power analysis in Suppl. Figure 2 showed that there was a good probability of finding a major QTL that explained 50\% of phenotypic variation if the year 2 estimate of $H^{2}$ for bioavailability was accurate (heritability measured here is only within the ferritin experimental set up). It is possible that the lack of clear major QTL for absorption could be a result of large field fertility effects within trials which were not accounted for here. However, given the trait distribution and transgressive segregation (Figure 1), we think it is most likely that Fe bioavailability is controlled by multiple loci of small effect, most of which were not detectable here. This was supported by the power analysis (Suppl. Figure 2) that showed there was low probability of finding minor QTL (percentage variation explained $=5 \%$ ) at either of the different year $H^{2}$ estimates, which would also explain to why we found no consistent loci across years. For future work with this population, we would recommend increasing the number of MAGIC individuals used which would increase the probability of finding consistent minor QTL across multiple environments. To the best of our knowledge, this is the largest single trial to date to measure and map bioavailability in wheat, and the absence of major QTL in a very diverse population, representing a large percentage of UK polymorphism, provides some insight into why progress in mapping and breeding for bioavailability has been slow. Finally, we note that there was also no correlation between Fe concentration and bioavailability in either year, no QTLs co-locate between the traits, and the QTL profiles (Figure 3) of the two traits are completely unrelated. This suggests that breeders will have to select not only for Fe levels directly in grains, but also increased bioavailability.

The role which phytate plays in Fe absorption is often highlighted in the literature, as nearly $2 / 3$ of the $\mathrm{Fe}$ in the grain is thought to be bound to phytate [21]. However, when direct measurements of phytate, $\mathrm{Fe}$ levels and absorption were all measured on the same samples, phytate levels do not explain the variation seen in the ability of Fe to be absorbed by the Caco- 2 cells. This suggests that other factors 
such as polyphenols or other yet identified components may be more important in increasing $\mathrm{Fe}$ absorption and not phytate per se. Although the phytate: Fe molar ratio was high for the samples measured which might be why no significant correlation was identified. One major problem with attempting to increase the bioavailability of Fe in wheat is that many of the phenolic acids which have been found to promote the absorption of iron, mainly from beans, do not appear to be produced in wheat [19,50-52]. It is unknown at this time if wheat cannot make these compounds or if other factors are needed for induction of their production. At the very least, we have not detected any simple explanations for the variation in bioavailability observed here, which is further consistent with it being a complex multi-genic trait.

Finally, as only whole grains were tested in this study, it would be interesting to understand phenotypic variation present in other portions of the grain and if the same QTL can be identified for both increasing Fe levels and absorption in the endosperm and germ. Recent studies in maize have shown that the germ itself can be a major inhibitory portion of the grain for Fe absorption and thus fortification of the endosperm which has been done by transgenic means in wheat might be a viable route to increase bioavailable iron [15,27]. Fe levels and phenolic acids are thought to be low in the endosperm, suggesting that the data collected to date will not help increase Fe absorption in white breads [52]. As $70 \%$ of the current consumption of bread is white bread and not wholemeal which would contain the phenolic acids, increasing absorption in this fraction might be more important than in wholegrain/wholemeal bread.

In conclusion, the large amount of between year variation for both traits and their underlying QTL, the absence of any correlation between Fe concentration and bioavailability, and the lack of major QTL for bioavailability, all highlight why little genetic progress has been made in addressing anemia from cereal based diets. Our results suggest that conventional breeding progress may be best achieved by focusing on iron bioavailability, rather than Fe concentration, and that results will be achieved incrementally via recurrent selection, (enhanced by genomic prediction) rather than by rapid deployment of a small number of major QTL alleles. Otherwise, traditional breeding might not fully address the issue of low Fe absorption in bread wheat.

\section{Declarations:}

Ethics approval and consent to participate: n/a

Consent for publication: $\mathrm{n} / \mathrm{a}$

Availability of data and materials: Wheat lines used in this publication are freely available upon a signed MTA from https://www.niab.com/research/agricultural-crop-research/resources/niab-magicpopulation-resources 
439 Competing interests: no competing interests.

440 Funding: This work was supported by BBSRC / ISCF Agri-Food Technology Seeding Catalyst Award $441 \quad 2017-18$

442 Authors' contributions: MM and RG performed the experiments, TW analyzed the data, and MM and 443 TW wrote the paper. All authors edited the paper and are happy with the submission.

444 Acknowledgements: n/a

445 
447 1. De Benoist B, World Health Organization., Centers for Disease Control and Prevention (U.S.).

448 Worldwide prevalence of anaemia 1993-2005 of: WHO Global Database of anaemia. World Health 449 Organization; 2008.

450 2. Zimmermann MB, Chaouki N, Hurrell RF. Iron deficiency due to consumption of a habitual diet low in bioavailable iron: a longitudinal cohort study in Moroccan children. Am J Clin Nutr. 2005;81:115-21.

3. Hurrell R. How to ensure adequate iron absorption from iron-fortified food. Nutr Rev. 2002;60:S7454 15; discussion S43.

4. Public Health England. National Diet and Nutrition Survey: results from Years 1 to 4 (combined) of the rolling programme for 2008 and 2009 to 2011 and 2012 - Publications - GOV.UK.

5. Federation of Bakers. Other industry data - Federation of Bakers [Internet]. [cited 2019 Oct 28]. Available from: https://www.fob.uk.com/about-the-bread-industry/industry-facts/other-industry-data/

6. Hurrell R, Ranum P, De Pee S, Biebinger R, Hulthen L, Johnson Q, et al. Revised recommendations for iron fortification of wheat flour and an evaluation of the expected impact of Current national wheat flour fortification programs. Food Nutr Bull. 2010;31.

7. Funk, Cary; Rainie, Lee; Page D. Public and Scientists' Views on Science and Society | Pew Research Center. 2015.

8. Rousselière $\mathrm{D}$, Rousselière $\mathrm{S}$. Is biotechnology (more) acceptable when it enables a reduction in phytosanitary treatments? A European comparison of the acceptability of transgenesis and cisgenesis. PLoS One. Public Library of Science; 2017;12.

467 9. Cui K, Shoemaker SP. Public perception of genetically-modified (GM) food: A Nationwide 468 Chinese Consumer Study. npj Sci Food. Springer Science and Business Media LLC; 2018;2.

10. Oury FX, Leenhardt F, Rémésy C, Chanliaud E, Duperrier B, Balfourier F, et al. Genetic variability and stability of grain magnesium, zinc and iron concentrations in bread wheat. Eur $\mathrm{J}$ Agron. 2006;25:177-85.

11. Zhao FJ, Su YH, Dunham SJ, Rakszegi M, Bedo Z, McGrath SP, et al. Variation in mineral micronutrient concentrations in grain of wheat lines of diverse origin. J Cereal Sci. 2009;49:290-5.

12. Khokhar JS, King J, King IP, Young SD, Foulkes MJ, De Silva J, et al. Novel sources of variation in grain Zinc (Zn) concentration in bread wheat germplasm derived from Watkins landraces. Zhang A, editor. PLoS One. NLM (Medline); 2020;15:e0229107.

13. Crespo-Herrera LA, Velu G, Singh RP. Quantitative trait loci mapping reveals pleiotropic effect for grain iron and zinc concentrations in wheat: Pleiotropic QTL for grain iron and zinc in a synthetic hexaploid wheat. Ann Appl Biol. 2016;169:27-35.

14. Eagling T, Wawer AA, Shewry PR, Zhao F-J, Fairweather-Tait SJ. Iron Bioavailability in Two Commercial Cultivars of Wheat: Comparison between Wholegrain and White Flour and the Effects of Nicotianamine and 2'-Deoxymugineic Acid on Iron Uptake into Caco-2 Cells. J Agric Food Chem. 2014;62:10320-5. Physiol. 2017;174:2434-44. 
Iron and zinc concentrations in rice grains via the introduction of barley genes involved in phytosiderophore synthesis. Rice. 2008;1:100-8.

17. Johnson AAT, Kyriacou B, Callahan DL, Carruthers L, Stangoulis J, Lombi E, et al. Constitutive overexpression of the OsNAS gene family reveals single-gene strategies for effective iron- and zincbiofortification of rice endosperm. PLoS One. 2011;6.

18. Zheng L, Cheng Z, Ai C, Jiang X, Bei X, Zheng Y, et al. Nicotianamine, a novel enhancer of rice iron bioavailability to humans. PLoS One. 2010;5.

19. Hart JJ, Tako E, Glahn RP. Characterization of Polyphenol Effects on Inhibition and Promotion of Iron Uptake by Caco-2 Cells. J Agric Food Chem. American Chemical Society; 2017;65:3285-94.

20. Hallberg L, Rossander L, Skaanberg AB. Phytates and the inhibitory effect of bran on iron absorption in man. Am J Clin Nutr. Am J Clin Nutr; 1987;45:988-96.

21. De Brier N, Gomand S V, Donner E, Paterson D, Smolders E, Delcour JA, et al. Element

distribution and iron speciation in mature wheat grains (Triticum aestivum L.) using synchrotron Xray fluorescence microscopy mapping and X-ray absorption near-edge structure (XANES) imaging. Plant Cell Environ. 2016;39:1835-47.

22. Jin F, Frohman C, Thannhauser TW, Welch RM, Glahn RP. Effects of ascorbic acid, phytic acid and tannic acid on iron bioavailability from reconstituted ferritin measured by an in vitro digestionCaco-2 cell model. Br J Nutr. Br J Nutr; 2009;101:972-81.

23. Engle-Stone R, Yeung A, Welch R, Glahn R. Meat and ascorbic acid can promote Fe availability from Fe-phytate but not from Fe-tannic acid complexes. J Agric Food Chem. 2005;53:10276-84.

24. Anson NM, Van Den Berg R, Havenaar R, Bast A, Haenen GRMM. Ferulic acid from aleurone determines the antioxidant potency of wheat grain (Triticum aestivum L.). J Agric Food Chem. American Chemical Society; 2008;56:5589-94.

25. Kim KH, Tsao R, Yang R, Cui SW. Phenolic acid profiles and antioxidant activities of wheat bran extracts and the effect of hydrolysis conditions. Food Chem. Elsevier; 2006;95:466-73.

26. Boukid F, Dall'Asta M, Bresciani L, Mena P, Del Rio D, Calani L, et al. Phenolic profile and antioxidant capacity of landraces, old and modern Tunisian durum wheat. Eur Food Res Technol. Springer Verlag; 2019;245:73-82.

27. Glahn R, Tako E, Gore MA. The germ fraction inhibits iron bioavailability of maize:

Identification of an approach to enhance maize nutritional quality via processing and breeding. Nutrients. MDPI AG; 2019;11.

28. Mackay IJ, Bansept-Basler P, Barber T, Bentley AR, Cockram J, Gosman N, et al. An eightparent multiparent advanced generation inter-cross population for winter-sown wheat: creation, properties, and validation. G3. 2014;4:1603-10.

29. Glahn RP, Lee OA, Yeung A, Goldman MI, Miller DD. Caco-2 Cell Ferritin Formation Predicts Nonradiolabeled Food Iron Availability in an In Vitro Digestion/Caco-2 Cell Culture Model. J Nutr. American Society for Nutrition; 1998;128:1555-61.

30. Cullis BR, Smith AB, Coombes NE. On the design of early generation variety trials with correlated data. J Agric Biol Environ Stat. 2006;11:381-93.

527 31. Computing RF for S. R Core Team (2018). R: A language and environment for statistical computing [Internet]. 2018. Available from: https://www.r-project.org/ 
wheat genomic diversity using a high-density 90000 single nucleotide polymorphism array. Plant Biotechnol J. Blackwell Publishing Ltd; 2014;12:787-96.

33. Gardner KA, Wittern LM, Mackay IJ. A highly recombined, high-density, eight-founder wheat MAGIC map reveals extensive segregation distortion and genomic locations of introgression segments. Plant Biotechnol J. Blackwell Publishing Ltd; 2016;14:1406-17.

34. Bates D, Mächler M, Bolker B, Walker S. Fitting Linear Mixed-Effects Models Using lme4. J Stat Softw. 2015;67.

35. Huang BE, George AW. R/mpMap: a computational platform for the genetic analysis of recombinant inbred lines. Bioinformatics. 2011;27:727-9.

36. Bouis HE, Hotz C, McClafferty B, Meenakshi J V., Pfeiffer WH. Biofortification: A new tool to reduce micronutrient malnutrition. Food Nutr Bull. 2011;32.

37. Bouis HE, Saltzman A. Improving nutrition through biofortification: A review of evidence from HarvestPlus, 2003 through 2016. Glob. Food Sec. Elsevier B.V.; 2017. p. 49-58.

38. Omari R, Zotor F, Tagwireyi J, Lokosang L. Advocacy for scaling up biofortified crops for improved micronutrient status in Africa: Approaches, achievements, challenges and lessons. Proc Nutr Soc. Cambridge University Press; 2019;

39. Ludwig Y, Slamet-Loedin IH. Genetic biofortification to enrich rice and wheat grain iron: From genes to product. Front. Plant Sci. Frontiers Media S.A.; 2019.

40. Connorton JM, Balk J. Iron Biofortification of Staple Crops: Lessons and Challenges in Plant Genetics. Plant Cell Physiol. Oxford University Press (OUP); 2019;

41. Velu G, Crespo Herrera L, Guzman C, Huerta J, Payne T, Singh RP. Assessing genetic diversity to breed competitive biofortified wheat with enhanced grain $\mathrm{Zn}$ and Fe concentrations. Front Plant Sci. Frontiers Media S.A.; 2019;9.

42. Dias DM, Kolba N, Binyamin D, Ziv O, Nutti MR, Martino HSD, et al. Iron biofortified carioca bean (phaseolus vulgaris L.) - based brazilian diet delivers more absorbable iron and affects the gut microbiota in vivo (gallus gallus). Nutrients. MDPI AG; 2018;10.

43. Garg M, Sharma N, Sharma S, Kapoor P, Kumar A, Chunduri V, et al. Biofortified Crops Generated by Breeding, Agronomy, and Transgenic Approaches Are Improving Lives of Millions of People around the World. Front Nutr. Frontiers Media SA; 2018;5.

44. Anderson GJ. Iron Biofortification: Who Gives a Bean? J Nutr. NLM (Medline); 2020;150:28412.

45. Glahn RP, Wiesinger JA, Lung'aho MG. Iron Concentrations in Biofortified Beans and Nonbiofortified Marketplace Varieties in East Africa Are Similar. J Nutr. NLM (Medline); 2020;150:3013-23.

46. Tiwari VK, Rawat N, Chhuneja P, Neelam K, Aggarwal R, Randhawa GS, et al. Mapping of Quantitative Trait Loci for Grain Iron and Zinc Concentration in Diploid A Genome Wheat. J Hered. 2009;100:771-6.

47. Arora S, Cheema J, Poland J, Uauy C, Chhuneja P. Genome-wide association mapping of grain micronutrients concentration in aegilops tauschii. Front Plant Sci. Frontiers Media S.A.; 2019;10.

48. Velu G, Singh R, Huerta-Espino J, Peña J, Ortiz-Monasterio I. Breeding for Enhanced Zinc and Iron Concentration in CIMMYT Spring Wheat Germplasm. 2010. 
571 49. Tiwari C, Wallwork H, Arun B, Mishra VK, Velu G, Stangoulis J, et al. Molecular mapping of 572 quantitative trait loci for zinc, iron and protein content in the grains of hexaploid wheat. Euphytica. $573 \quad 2015 ; 207: 563-70$.

574 50. Hart JJ, Tako E, Kochian L V., Glahn RP. Identification of Black Bean ( Phaseolus vulgaris L.) 575 Polyphenols That Inhibit and Promote Iron Uptake by Caco-2 Cells. J Agric Food Chem.

576 2015;63:5950-6.

577 51. Tako E, Beebe SE, Reed S, Hart JJ, Glahn RP. Polyphenolic compounds appear to limit the 578 nutritional benefit of biofortified higher iron black bean (Phaseolus vulgaris L.). Nutr J. BioMed 579 Central Ltd.; 2014;13.

580 52. Zhou K, Su L, Yu L. Phytochemicals and antioxidant properties in wheat bran. J Agric Food 581 Chem. American Chemical Society ; 2004;52:6108-14.

582 53. Wei T, Simko V. corrplot. 2017. 
586 Table 1 - Sample number $(n)$, overall trait means $(\mu)$ and standard deviation $(\sigma)$ for the population individuals used in QTL mapping of Fe concentration and bioavailability. The observed means (Fe concentration) or best linear unbiased predictions (bioavailability) are shown for each of the MAGIC founder lines. For each year, the average standard error of differences between lines was calculated for bioavailability during the model fitting stage (average SED).

\begin{tabular}{ccccccccccccc}
\hline Trait & Year & $n$ & $\mu$ & $\sigma$ & $\mathrm{Al}$ & $\mathrm{Br}$ & $\mathrm{Cl}$ & $\mathrm{He}$ & $\mathrm{Ri}$ & $\mathrm{Ro}$ & So & $\mathrm{Xi}$ \\
\hline Bioavailability & 1 & 235 & 5.5 & 1.0 & 7.5 & 6.3 & 7.9 & 6.7 & 7.5 & 5.2 & 5.8 & 6.3 \\
& Average SED $=1.49$ & & & & & & & & & & \\
Bioavailability & 2 & 284 & 10.9 & 4.1 & - & - & 9.4 & - & - & 11.9 & - & - \\
& Average SED $=1.53$ & & & & & & & & & & \\
Fe concentration & 1 & 237 & 32.8 & 4.0 & 28.2 & 34.6 & 33.7 & 33.5 & 31.3 & 37.2 & 34.7 & 33.8 \\
Fe concentration & 2 & 284 & 32.3 & 4.5 & - & - & 21.7 & - & - & 29.2 & - & - \\
\hline
\end{tabular}

Trait units - Bioavailability: Ferritin / Protein (ng / mg). Fe concentration: Fe (ppm).

$\mathrm{Al}=$ Alchemy, $\mathrm{Br}=$ Brompton, $\mathrm{Cl}=$ Claire, $\mathrm{He}=$ Hereward, $\mathrm{Ri}=$ Rialto, $\mathrm{Ro}=$ Robigus, $\mathrm{So}=$ Soissons, $\mathrm{Xi}=\mathrm{Xi19}$.

591

592

593

594

595

596

597

598 
Table 2 - Candidate QTL for Fe concentration and bioavailability identified through interval (IM) and composite interval mapping (CIM) using mpMap [35]. For each QTL, the table shows the mapped chromosome (Chr) and location (Pos), parental effects with the founder Xi19 used as a baseline, the flanking array markers, the Wald test statistic (Wald) and associated $P$ value significance thresholds. The $P$ values expressed to - log 10 and the percentage phenotypic variation

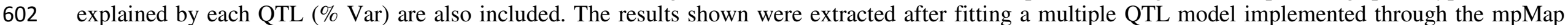
603 function 'fit()'.

\begin{tabular}{|c|c|c|c|c|c|c|c|c|c|c|c|c|c|c|c|}
\hline Year & Method & Flanking Markers (left - right) & $\mathrm{Chr}$ & Pos $(\mathrm{cM})$ & $\mathrm{Al}$ & $\mathrm{Br}$ & $\mathrm{Cl}$ & $\mathrm{He}$ & $\mathrm{Ri}$ & Ro & So & $\mathrm{Xi}$ & Wald & $-\log 10$ & $\%$ Var \\
\hline \multicolumn{16}{|c|}{ Fe concentration } \\
\hline 1 & IM & RAC875_c67311_429 - RFL_Contig4718_1323 & $2 \mathrm{~B}$ & 216.09 & -15.7 & -33.9 & -7.0 & -31.8 & -14.5 & -19.6 & -19.3 & 0 & $28.08^{* * *}$ & 3.7 & 7.7 \\
\hline 1 & CIM & Kukri_c148_1484 - BobWhite_c1149_539 & $2 \mathrm{~B}$ & 29.5 & -0.2 & -1.9 & -1.1 & -0.1 & -2.6 & -2.0 & -2.8 & 0 & $20.35^{* *}$ & 2.3 & 4.8 \\
\hline 1 & CIM & RFL_Contig4718_1323-BS00092235_51 & $2 \mathrm{~B}$ & 217.5 & -3.1 & -9.8 & -1.6 & -19.5 & 3.3 & -8.2 & -8.1 & 0 & $43.72^{* * *}$ & 6.6 & 7.4 \\
\hline 1 & $\mathrm{IM}$ & BS00039852_51 - RAC875_c8313_72 & $3 \mathrm{D}$ & 46.84 & 5.0 & 0.6 & -1.5 & 3.9 & -8.9 & -2.8 & -2.5 & 0 & $18.75^{* *}$ & 2.1 & 7.7 \\
\hline 1 & CIM & BobWhite_c42020_456-Ex_c4296_1270 & $3 \mathrm{D}$ & 180.63 & -3.2 & 3.3 & 1.4 & -1.5 & 2.8 & 0.2 & 3.4 & 0 & $23.62^{* *}$ & 2.9 & 3.9 \\
\hline 1 & $\mathrm{IM}$ & tplb0023j07_1091 - RAC875_c63933_184 & $5 \mathrm{D}$ & 175.5 & 0.0 & -0.5 & -1.8 & 1.4 & -2.0 & -1.8 & 0.8 & 0 & $16.66^{*}$ & 1.7 & 7.7 \\
\hline 1 & CIM & BS00055493_51 - D_GB5Y7FA02JRQ1I_101 & $5 \mathrm{D}$ & 199.08 & -1.3 & -0.8 & -6.5 & -0.6 & -6.7 & -2.8 & -1.7 & 0 & $37.9^{* * *}$ & 5.5 & 6.5 \\
\hline 1 & IM & TA004558_1018 - Ra_c14408_576 & $6 \mathrm{~A}$ & 128.93 & -3.2 & -2.0 & -1.1 & -2.0 & -4.0 & -1.4 & -0.7 & 0 & $22.86^{* *}$ & 2.7 & 8.0 \\
\hline 1 & CIM & TA004558_1018 - Ra_c14408_576 & $6 \mathrm{~A}$ & 128.93 & -4.5 & -3.1 & -2.0 & -2.4 & -5.0 & -2.2 & -1.8 & 0 & $38.43^{* * *}$ & 5.6 & 8.0 \\
\hline 2 & $\mathrm{IM}$ & BS00012942_51 - Tdurum_contig42013_538 & $2 \mathrm{~A}$ & 252.8 & -0.4 & 0.3 & -2.9 & -2.1 & -1.2 & -4.0 & -4.1 & 0 & $26.08^{* * *}$ & 3.3 & 6.3 \\
\hline 2 & CIM & BS00012942_51 - Tdurum_contig42013_538 & $2 \mathrm{~A}$ & 252.8 & -0.6 & 0.2 & -2.9 & -2.2 & -1.3 & -4.0 & -4.2 & 0 & $26.01^{* * *}$ & 3.3 & 6.3 \\
\hline \multicolumn{16}{|c|}{ Fe bioavailability } \\
\hline 1 & $\mathrm{IM}$ & TA005289_1104 - IAAV3156 & $1 \mathrm{~A}$ & 167.31 & -0.2 & -0.8 & 0.5 & 0.5 & -0.5 & 1.2 & 0.8 & 0 & $16.61^{*}$ & 1.7 & 8.1 \\
\hline 1 & $\mathrm{IM}$ & BS00079088_51 - BS00065268_51 & $1 \mathrm{~A}$ & 193.67 & -0.4 & -2.1 & 1.6 & -1.3 & -0.1 & -0.9 & -0.6 & 0 & $17.38^{*}$ & 1.8 & 8.4 \\
\hline 1 & CIM & BS00065268_51 - Kukri_c310_1953 & $1 \mathrm{~A}$ & 195 & -0.6 & -2.8 & 2.6 & -1.1 & -0.6 & -0.4 & -0.2 & 0 & $36.65^{* * *}$ & 5.3 & 8.2 \\
\hline 1 & CIM & $\begin{array}{c}\text { wsnp_Ex_c35331_43499339 - } \\
\text { wsnp_JD_rep_c48914_33168544 }\end{array}$ & $2 \mathrm{~A}$ & 87.5 & -1.3 & 0.3 & -0.7 & -1.1 & -2.3 & -0.5 & -0.6 & 0 & $28.89^{* * *}$ & 3.8 & 5.8 \\
\hline 1 & CIM & BS00022498_51-RAC875_c1638_165 & $7 \mathrm{~B}$ & 72.41 & 0.3 & -1.2 & -1.7 & -0.1 & 0.0 & 0.3 & -0.1 & 0 & $25.29^{* * *}$ & 3.2 & 3.6 \\
\hline 2 & CIM & Excalibur_c12980_2392 - wsnp_Ra_c8771_14786376 & $2 \mathrm{~A}$ & 10.5 & -0.4 & 3.4 & 1.8 & -0.9 & 2.5 & 2.6 & 1.7 & 0 & $24.6^{* * *}$ & 3.0 & 4.5 \\
\hline 2 & CIM & BS00084904_51-Excalibur_c100336_106 & $4 \mathrm{~B}$ & 55.7 & -0.5 & -2.2 & -0.6 & -1.4 & 0.4 & -3.0 & 1.4 & 0 & $24.2^{* * *}$ & 3.0 & 4.4 \\
\hline
\end{tabular}

$\mathrm{Al}=$ Alchemy, $\mathrm{Br}=$ Brompton, $\mathrm{Cl}=$ Claire, $\mathrm{He}=$ Hereward, $\mathrm{Ri}=$ Rialto, Ro = Robigus, $\mathrm{So}=$ Soissons, $\mathrm{Xi}=\mathrm{Xi1}$. 
611

612

613

614

615

616

617

618

619

620

621

622

623

624

625

626

627

628

629

630

Table 3 - Candidate QTL identified through the IBS mapping. Only QTL with $-\log 10(P)$ values above the Bonferroni significance threshold are shown, which was estimated using population haplotype number. The chromosome the QTL was found on (Chr) and MAGIC genetic linkage map position (Pos) are shown for each QTL hit. The SNP effect represents the fixed effect from each IBS model fitted using lme4 in R [34]. The $P$ values were also adjusted using a false discovery rate (FDR) adjustment for total test number.

\begin{tabular}{|c|c|c|c|c|c|c|c|}
\hline Year & Marker & $\mathrm{Chr}$ & $\begin{array}{l}\text { Pos } \\
\text { (cM) }\end{array}$ & $\begin{array}{c}\text { FDR } \\
\text { adjusted } P\end{array}$ & $\begin{array}{c}\text { Bonf. } \\
\text { threshold }\end{array}$ & $-\log 10(P)$ & $\begin{array}{l}\text { SNP } \\
\text { effect }\end{array}$ \\
\hline \multicolumn{8}{|c|}{ Fe concentration } \\
\hline 1 & wsnp_Ex_rep_c67543_66165372 & $2 \mathrm{~B}$ & 220.7 & 0.1 & 3.68 & 3.95 & 1.14 \\
\hline 1 & BS00032035_51 & $5 \mathrm{D}$ & 181.1 & 0.06 & 3.68 & 4.55 & 1.11 \\
\hline \multicolumn{8}{|c|}{ Bioavailability } \\
\hline 1 & Ra_c73292_443 & $5 \mathrm{~B}$ & 91.3 & 0.21 & 3.68 & 3.79 & -0.36 \\
\hline 2 & Excalibur_rep_c110303_320 & $2 \mathrm{~A}$ & 18 & 0.26 & 3.68 & 3.71 & -1.09 \\
\hline
\end{tabular}

12

13

8




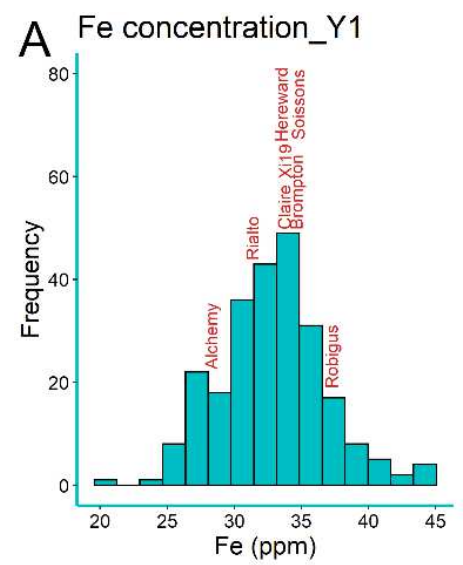

B Fe concentration_Y2
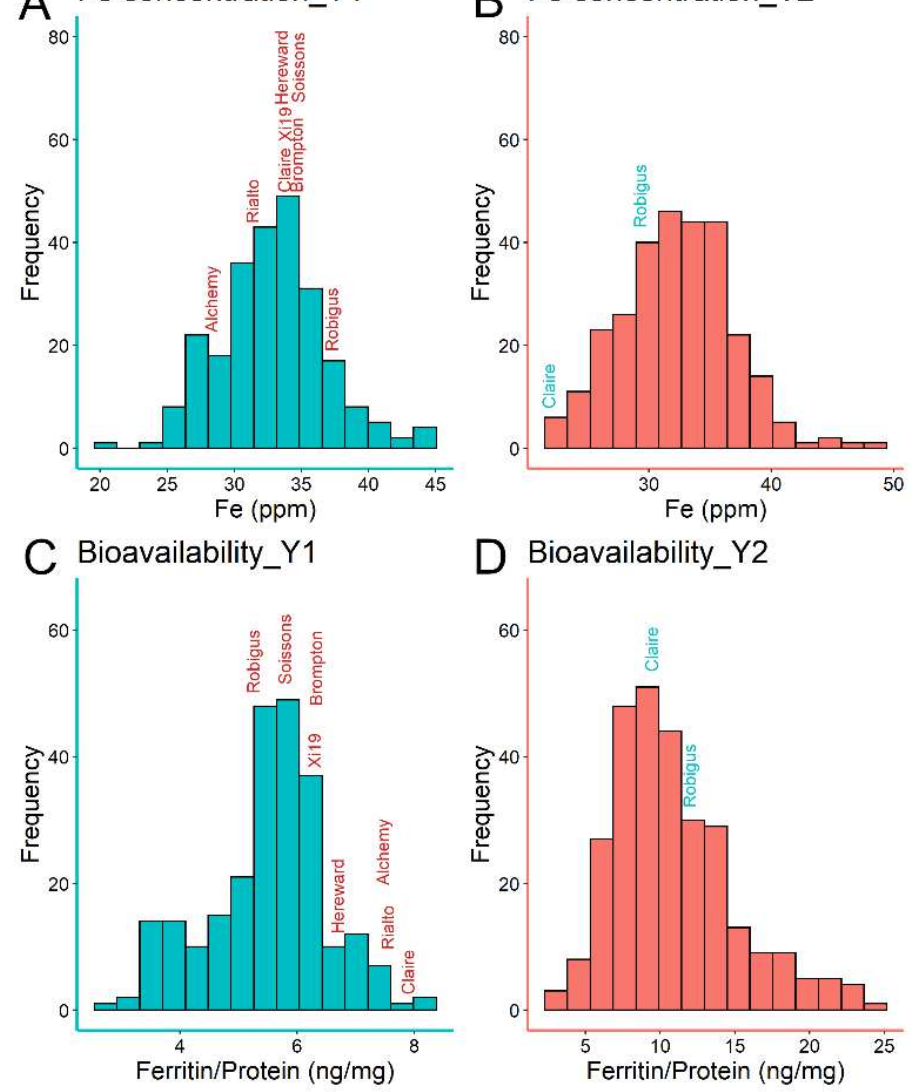

D Bioavailability_Y2
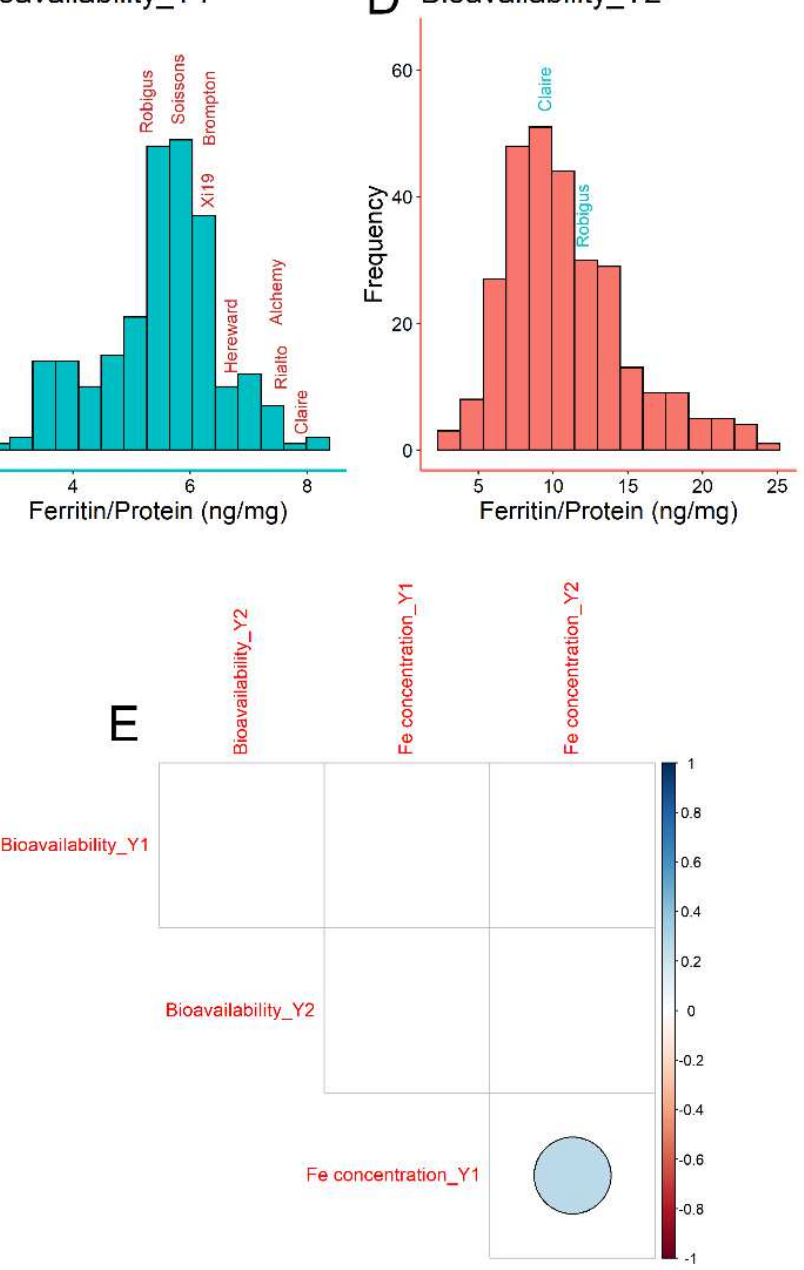

Figure 1 - Phenotype frequency plots for the four traits measured. Including the observed means from year 1 and year 2 for grain Fe concentration ( $\mathbf{A}$ and $\mathbf{B}$, respectively) and the corrected means (best linear unbiased predictions) for Fe bioavailability in year 1 and year 2 (C and $\mathbf{D}$, respectively). The MAGIC founder values for each trait are overlaid on each histogram, signified by a text label. In year 1 all founders were measured, while only two founders (Claire and Robigus) were measured in year 2. Also shown is a graphical correlation matrix for the bioavailability line means and the observed $\mathrm{Fe}$ concentration means from both years $(\mathbf{E})$. Correlations left blank signify that the $P$ value associated with the Pearson's correlation test was greater than $P=0.01$. The correlation matrix was plotted using the R package "corrplot" [53]. 

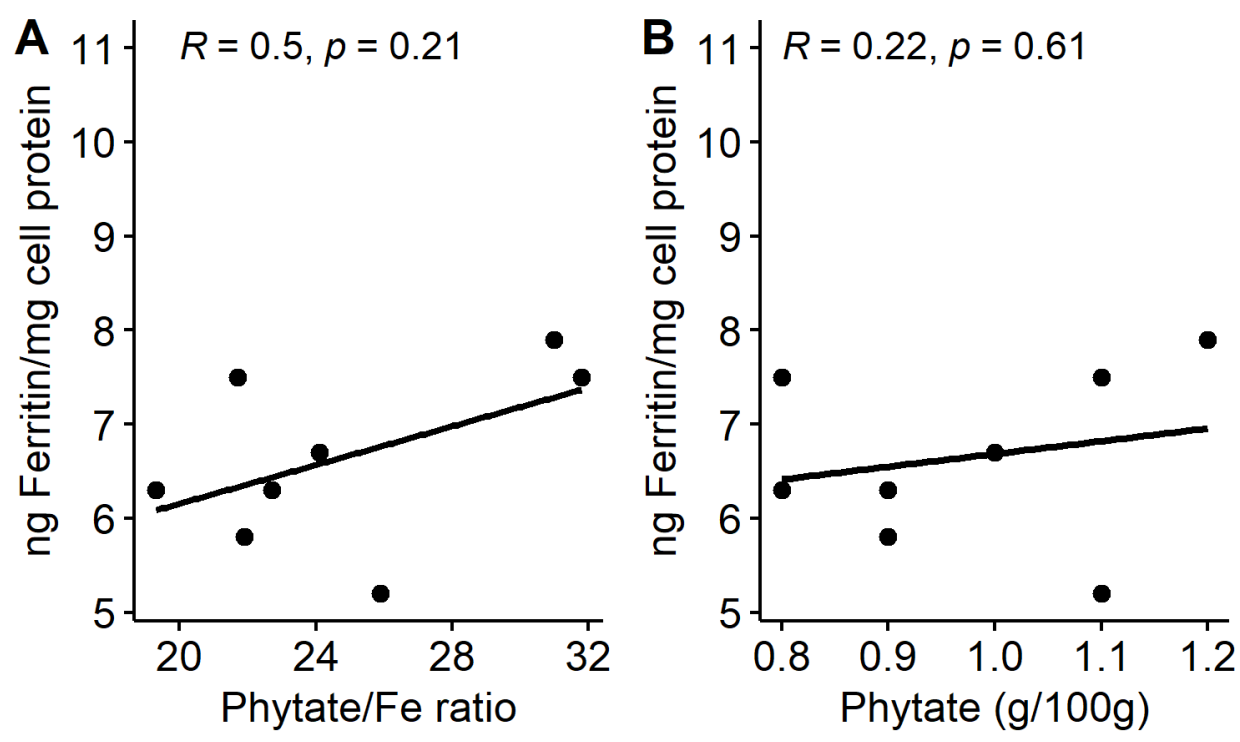

643 Figure 2-A: The average ferritin response to whole grains from each of the MAGIC founders compared 644 to the average ratio of phytic acid to Fe concentrations in the grain and its ability to be bound by ferritin. 645 B: The average ferritin response versus the amount of Phytate measured in the grain in the MAGIC 646 founders. All data shown was taken from year 1. 

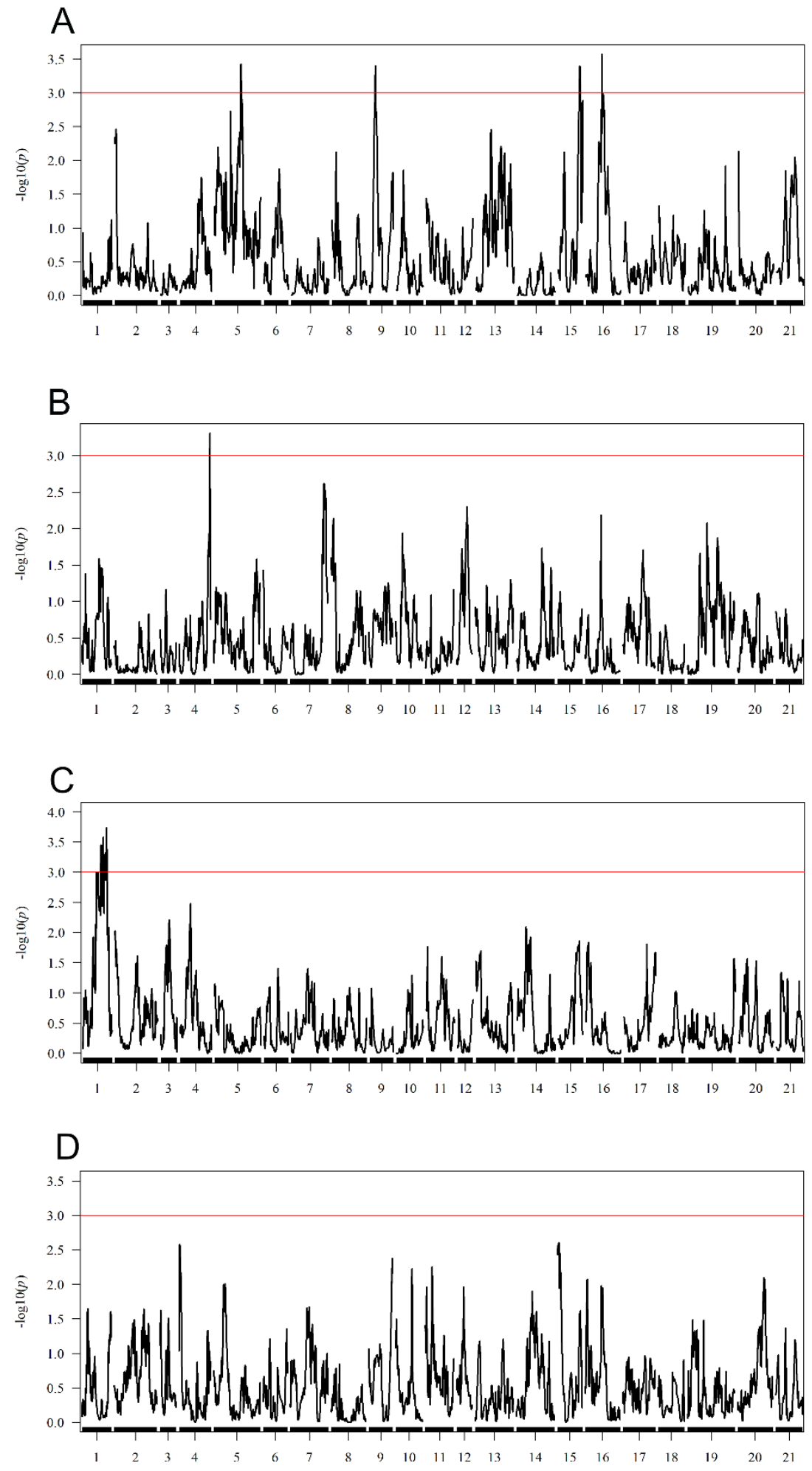

Chromosome

Figure 3 - Interval mapping profiles for the two traits measured across two years. A: Fe concentration in year 1. B: Fe concentration in year 2. C: Bioavailability in year 1. D: Bioavailability in year 2. For each plot the $-\log 10(p)$ values are shown across the 21 chromosomes of bread wheat. A $-\log 10(p)$ threshold of 3 is shown as a cut-off for significance. The results show the preliminary output from the interval mapping scan, before the mixed model fitting using 'fit()'. 


\section{Figures}
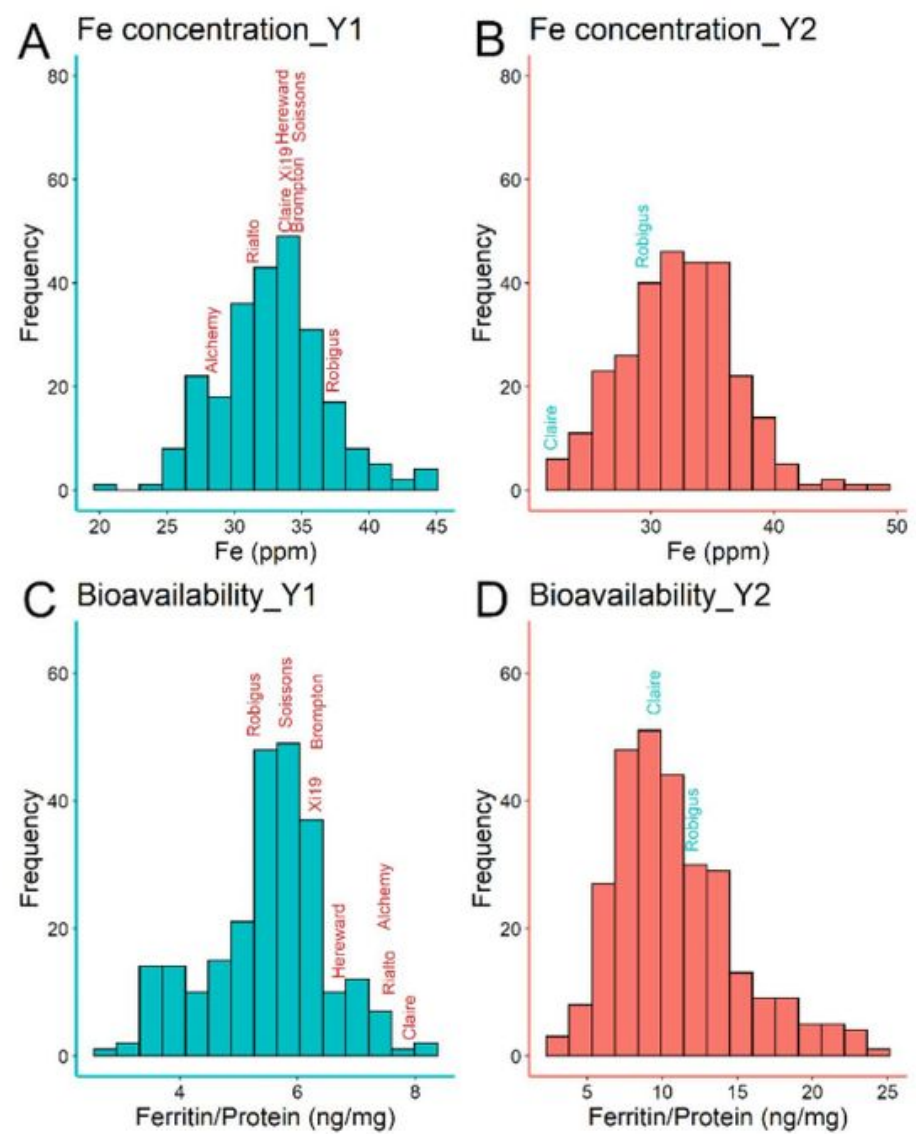

D Bioavailability_Y2
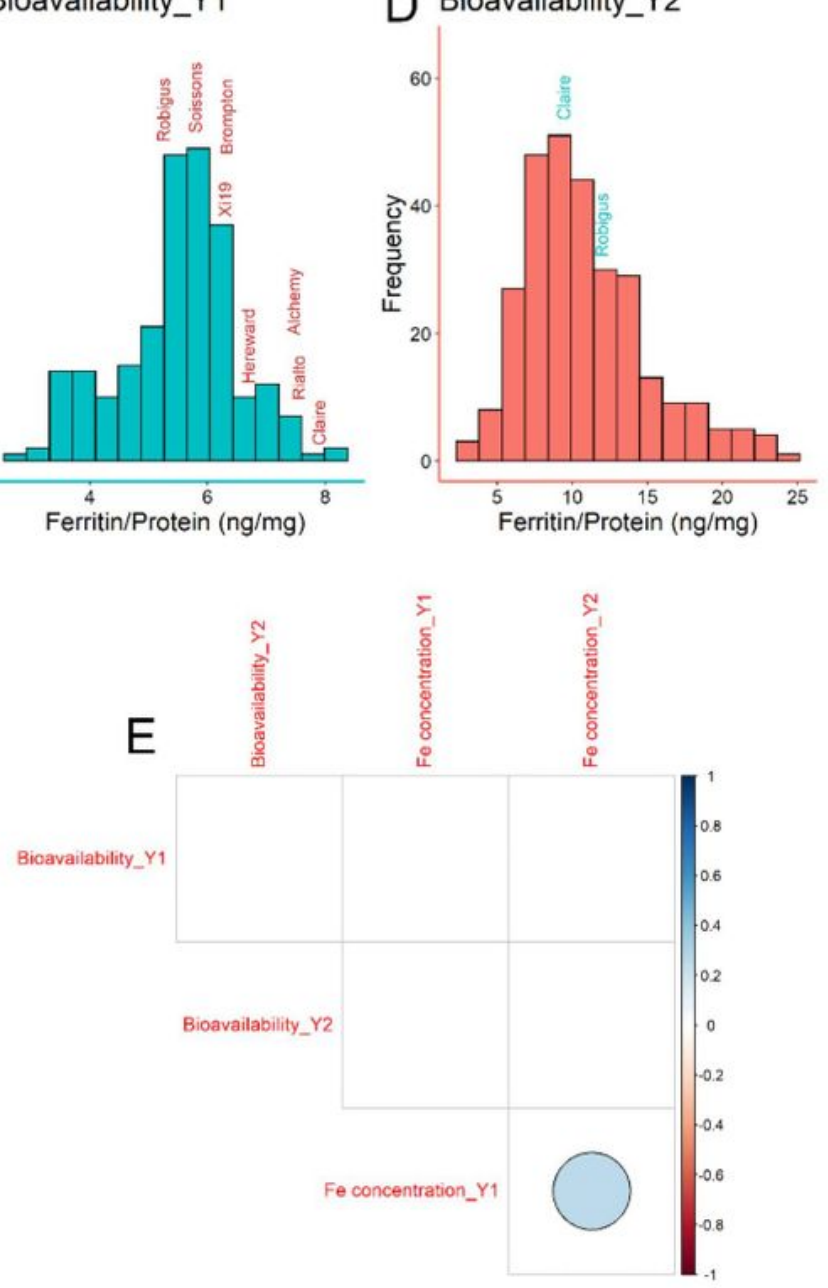

\section{Figure 1}

Phenotype frequency plots for the four traits measured. Including the observed means from year 1 and year 2 for grain Fe concentration ( $A$ and $B$, respectively) and the corrected means (best linear unbiased predictions) for Fe bioavailability in year 1 and year 2 ( $C$ and $D$, respectively). The MAGIC founder values 
for each trait are overlaid on each histogram, signified by a text label. In year 1 all founders were measured, while only two founders (Claire and Robigus) were measured in year 2. Also shown is a graphical correlation matrix for the bioavailability line means and the observed Fe concentration means from both years $(E)$. Correlations left blank signify that the $P$ value associated with the Pearson's correlation test was greater than $\mathrm{P}=0.01$. The correlation matrix was plotted using the $\mathrm{R}$ package "corrplot" [53].
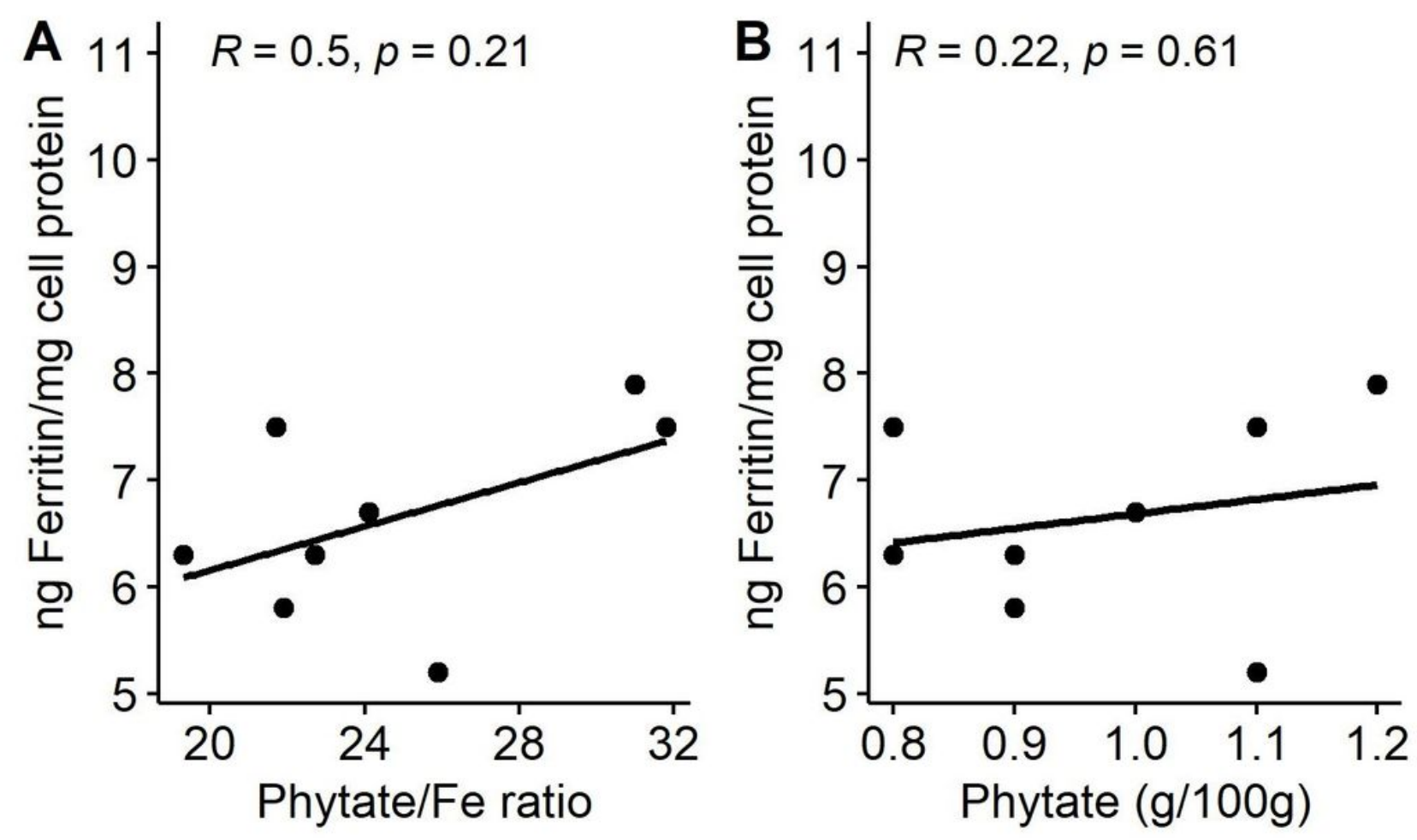

Figure 2

$A$ : The average ferritin response to whole grains from each of the MAGIC founders compared to the average ratio of phytic acid to Fe concentrations in the grain and its ability to be bound by ferritin. $\mathrm{B}$ : The average ferritin response versus the amount of Phytate measured in the grain in the MAGIC founders. All data shown was taken from year 1 . 

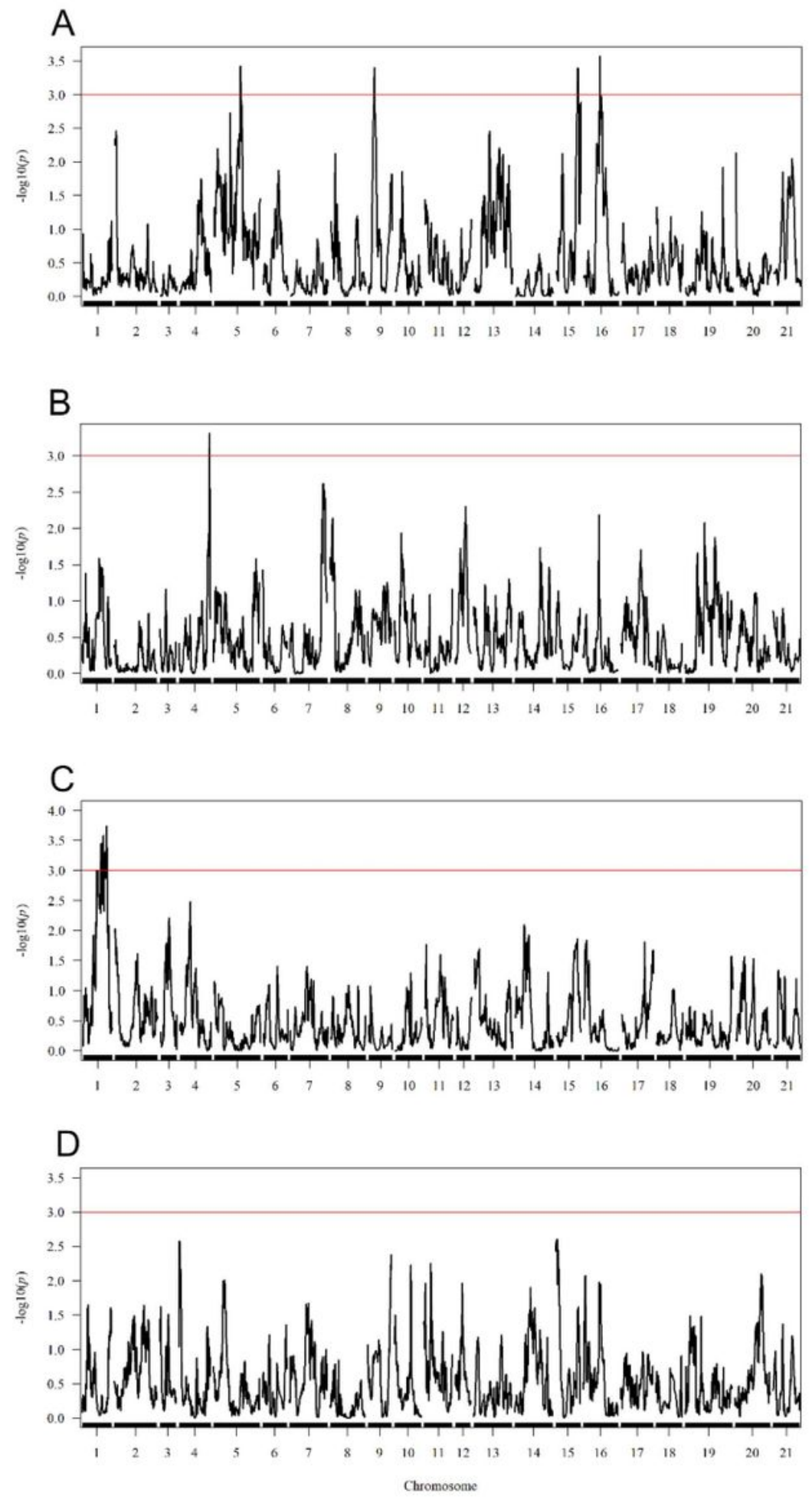

Figure 3

Interval mapping profiles for the two traits measured across two years. A: Fe concentration in year 1. B: Fe concentration in year 2. C: Bioavailability in year 1. D: Bioavailability in year 2. For each plot the - $\log 10$ (p) values are shown across the 21 chromosomes of bread wheat. A - $\log 10(p)$ threshold of 3 is shown as a cut-off for significance. The results show the preliminary output from the interval mapping scan, before the mixed model fitting using 'fit()'. 


\section{Supplementary Files}

This is a list of supplementary files associated with this preprint. Click to download.

- WrightetalBioavailpresubmissionSUPPLEMENTAL.pdf 